\title{
Korrekturosteotomie der fehlverheilten Monteggia-Verletzung mit einem Fixateur externe
}

\author{
Theddy F. Slongo'
}

\section{Zusammenfassung \\ Operationsziel}

Definitive Reposition des chronisch luxierten Radiuskopfs zur Verhinderung einer möglichen späteren Schädigung oder Funktionseinbuße.

\section{Indikationen}

Bei frischen Verletzungen immer.

Bei chronischer Luxation bei Beschwerden.

\section{Kontraindikationen}

Prinzipiell keine.

\section{Operationstechnik}

Die Operationstechnik hat sich prinzipiell nach der zu korrigierenden Fehlstellung oder Grundproblematik sowie dem zur Verfügung stehenden Instrumentarium und Implantat zu richten. Es gibt mehrere Techniken: alleinige angulierende Ulnaosteotomie (kurzes Intervall nach Unfall $<3$ Monate), angulierende und verlängernde Ulnaosteotomie (meist > 3 Monate nach Unfall); ein zusätzliches offenes Vorgehen ist selten notwendig bzw. sollte unterlassen werden.

\section{Postoperative Behandlung}

Abhängig vom Alter des Kinds und der gewählten Technik (Fixateur externe, Platte). Es sollte aber nach Möglichkeit immer eine gipsverbandfreie Nachbehandlung angestrebt werden (bessere Funktion).

\section{Ergebnisse}

Die Ergebnisse hängen von der Erfahrung des Operateurs, der Genauigkeit der Planung und der gewählten Technik ab. Eine intensive physiotherapeutische Nachbehandlung ist unumgänglich, häufig initial unter Einsatz einer Motorschiene. Korrekturverluste und Rezidive sind bekannt und nicht selten. Diese kommen bei ungenügender Stabilisierung oder Zentrierung zustande; aussagekräftige Zahlen gibt es nicht, doch die klinische Erfahrung bestätigt dies nur allzu oft.

\section{Schlüsselwörter}

Monteggia-Verletzung · Radiuskopfluxation • Bowing-Fraktur · Ulnaosteotomie · Fixateur externe $\cdot$ Ringfixateur $\cdot$ Ilisarov
Oper Orthop Traumatol 2008;20:435-49

DOI 10.1007/s00064-008-1414-8

${ }^{1}$ Abteilung für Pädiatrische Chirurgie, Universitätskinderklinik, Inselspital, Bern, Schweiz. 


\section{Correction Osteotomy of Neglected “Monteggia" Lesion with an External Fixator}

\section{Abstract \\ Objective}

Definite reduction of the dislocated radial head to prevent further damage and loss of function in case of a neglected radial head dislocation following Monteggia lesion. Three different operation methods are demonstrated.

\section{Indications}

A correction must be striven for in any fresh injury. The indication has to be made according to the complaints in chronic dislocations. A correction is very difficult and not always successful.

\section{Contraindications In principle, none.}

\section{Surgical Technique}

In principle, the surgical technique depends on the underlying disease and the kind of deformity, as well as on the instruments and implants available. There are different techniques: exclusive angulation osteotomy of the ulna (short interval after injury < 3 months), angulation osteotomy and additional lengthening of the ulna (mostly $>3$ months after injury); an open procedure is seldom necessary and should be avoided.

\section{Postoperative Management}

Depending of the child's age, the technique used and the implant chosen (external fixator, plate), a cast-free treatment should be striven for (better function).

\section{Results}

The results depend on the surgeon's experience, the accuracy of planning, and the technique chosen. An intensive postoperative physiotherapy is mandatory, frequently with initial application of CPM (continuous passive motion). Loss of correction and residual malalignments are well known and not rare. They occur mostly in instances of insufficient stability and centralization of the radial head, respectively. There are no evident numbers but this is confirmed by clinical experience.

\section{Key Words}

Monteggia lesion $\cdot$ Radial head dislocation $\cdot$ Bowing fracture · Ulna osteotomy · External fixator · Ring fixator · llizarov

\section{Vorbemerkungen}

Eine fehlverheilte Monteggia-Verletzung kann im Gegensatz zu Fehlstellungen im Schaftbereich häufig mit einer schweren Beeinträchtigung der Ellbogenfunktion einhergehen und lässt sich oft nur schwer oder gar nicht korrigieren.

Prinzipiell gibt es drei Ursachen [2, 4]:

1. Die Verletzung wird übersehen, am häufigsten, weil das Ellbogengelenk nicht mitgeröntgt oder eine unvollständige Fraktur der Ulna nicht erkannt wurde.

2. Die Verletzung wird nicht korrekt ruhig gestellt. Dadurch kommt es zur Redislokation des Radiuskopfs. Dies ist vorwiegend nach Bowing-Frakturen zu beobachten.

3. Eine erkannte Verletzung wird ignoriert. Dieses Verhalten ist leider nicht selten und absolut unverständlich. Noch dazu kommen solche Patienten oft sehr spät in Behandlung.

Die Indikation zur Korrektur hängt von verschiedenen Faktoren ab [1, 4, 7]. Oft führt die Verletzung anfangs kaum zu klinischen Problemen. In dieser Situation sollten Kind und Eltern über mögliche Folgen aufgeklärt werden (eingeschränkte Flexion und/oder Extension,
Probleme mit der Umwendbewegung, Irritation des Nervus ulnaris).

In Abhängigkeit vom Ausmaß der Radiuskopfluxation und - noch wichtiger - von der Zeitspanne seit der Verletzung ist dieses Ziel jedoch nicht in jedem Fall zu erreichen. Diese Erkenntnis muss man sich bei der Indikationsstellung immer vor Augen halten, denn eine falsche Einschätzung der Behandlungsmöglichkeiten führt nicht selten zu einer Verschlechterung der Situation.

Oft muss man sich mit einem Teilziel, nämlich Beschwerdefreiheit, begnügen. Das gilt besonders bei veralteten, lange vernachlässigten oder übersehenen Verletzungen. Es ist wichtig, mit den Eltern darüber zu sprechen. Gelegentlich kann es besser sein, in dieser Situation auch einmal Nein zu sagen.

Nicht jede Fehlstellung muss unbedingt korrigiert werden, gelegentlich kann der Spontanzustand, der sich eingestellt hat, funktionell und von den Beschwerden her besser sein als der Zustand nach einer versuchten oder fehlgeschlagenen Korrektur.

Folgende Faktoren spielen bei der Indikationsstellung eine Rolle: 
1. Zustand des proximalen Radioulnargelenks (Abbildung 1a): Bei Luxation des Radiuskopfs verändert sich die Konkavität des proximalen Radioulnargelenks schnell, es verliert seine Konkavität und wird konvex. Das wird oft nicht realisiert. In diesen Fällen ist eine korrekte Einstellung des Kopfs nicht mehr möglich, und es verbleibt eine Subluxationsstellung. Dieses Kriterium ist bereits auf einer korrekten seitlichen Röntgenaufnahme des Ellbogens zu sehen, deutlicher erkennt man die Veränderungen mit Hilfe einer Feinschicht-Computertomographie (CT). Ist das Gelenk noch deutlich sichtbar und fehlt eine wesentliche dorsale Dislokation des Radius, kann es mit einem Fixateur direkt korrekt eingestellt werden; d.h., nach der Ulnaosteotomie wird über direkte Manipulation des Fixateurs durch die Angulation der Ulna die Reposition des Radiuskopfs erzwungen. Ist das Gelenk dagegen ,aufgebraucht“, so ist eine perfekte Einstellung weder offen noch geschlossen möglich, und die Therapie besteht in einer Ulnaverlängerung.

2. Ausmaß der Dislokation des Radiuskopfs: Überragt der Radiuskopf das Capitulum humeri um $<5 \mathrm{~mm}$, ist eine akute Korrektur noch erfolgreich möglich. Bei größerer Dislokation kann nur eine kontinuierliche Verlängerung der Ulna mit zusätzlicher temporärer Fixierung des distalen Radius an die Ulna zum Erfolg führen.
3. Deformation des luxierten Radiuskopfs (Abbildung 1b): Je länger die Dislokation besteht und je ausgeprägter sie ist, umso größer ist die Deformation des Radiuskopfs. Hier kann ebenfalls nur eine Ulnaverlängerung Erfolg bringen. Es ist wichtig, darauf hinzuweisen, dass es in dieser Situation sinnlos ist, eine Rekonstruktion offen vorzunehmen.

4. Alter des Patienten und der Verletzung: Je älter das Kind ist und je länger die Dislokation besteht, umso zurückhaltender sollte man mit der Indikationsstellung sein. Nur bei großer Erfahrung mit Verlängerungen und Korrekturen sollte man sich an diese $\mathrm{Pa}$ tienten wagen.

5. Verbliebene Deformation der Ulna: Da eine der Hauptursachen der sich langsam entwickelnden Dislokation des Radiuskopfs die Deformation der Ulna ist, besonders bei nicht realisierter Bowing-Fraktur, muss diese beseitigt werden.

Kaum eine andere Fehlstellung hängt so stark von einem guten Behandlungsprinzip ab. Verfügt man nur über eine Strategie oder eine Methode, wird man die vielfältigen Probleme nicht zufriedenstellend lösen können. Wie schon mehrmals betont, spielt auch hier die Zeitspanne eine wichtige Rolle: Während frische Verletzungen noch mit relativ wenig Aufwand behandelt werden können, lassen sich langfristig veraltete Läsionen unserer Meinung nach nur noch mit einem sog. Ringfixateur behandeln $[3,10]$.

\begin{abstract}
Abbildungen 1a und $1 \mathrm{~b}$
a) Zustand des proximalen Radioulnargelenks; die Pfeile markieren die nicht mehr sichtbare Sklerose des ehemaligen Gelenks, Situation 8 Monate nach Unfall.

b) Schwer deformierter Radiuskopf mit der Unmöglichkeit einer Einstellung.
\end{abstract}
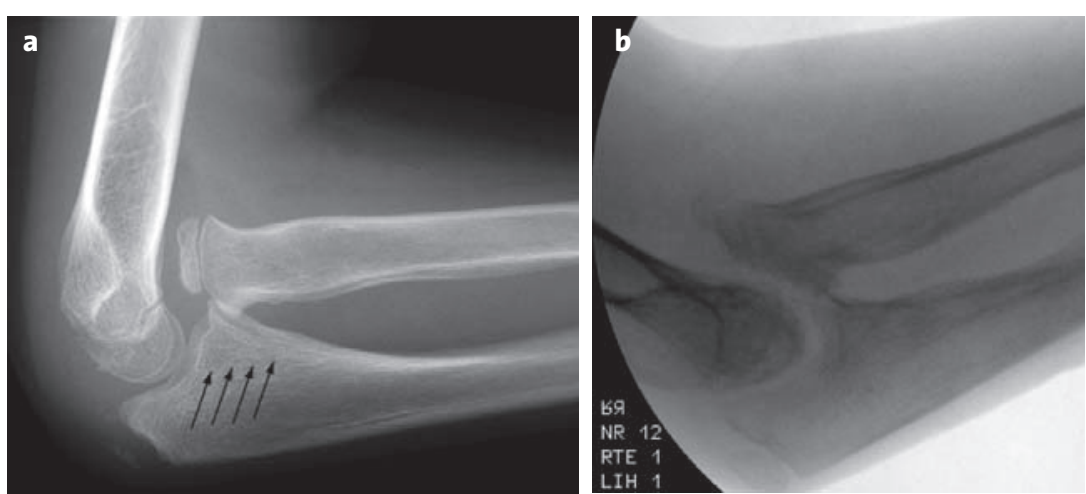


\section{Operationsprinzip und -ziel}

Das Prinzip der Operation besteht zum einen darin, die Fehlstellung der Ulna zu beseitigen; dies ist nur über eine Ulnaosteotomie zu erreichen. Als zweiter Schritt muss bei einer dorsalen Dislokation des Radiuskopfs $\geq 5 \mathrm{~mm}$ eine gleichzeitige Ulnaverlängerung durchgeführt werden, vorteilhafterweise mit einem Fixateur externe (Typ: ,tube-to-tube“ oder Ringfixateur). Ziel ist eine optimale Wiederherstellung der funktionellen und anatomischen Verhältnisse des Ellbogen-

\section{Vorteile}

- Dreidimensionale Korrekturmöglichkeit.

- Spontanes Einstellen des Radiuskopfs in korrekter Position.

- Verlängerung und Angulation der Ulna.

- Nachkorrektur möglich.

- Stabile Versorgung.

- Funktionelle Nachbehandlung.

- Intraoperatives Resultat auch postoperativ praktisch garantiert.

\section{Nachteile}

- Allgemeine Nachteile bei Verwendung eines Fixateur externe.

- Schmerz.

- Infektion der Pineintrittsstellen.

\section{Indikationen}

- Beeinträchtigung der Ellbogenfunktion in Flexion/ Extension oder Supination/Pronation.

- Schmerz, meist im Bereich des Nervus ulnaris.

- Kosmetische Beeinträchtigung.

\section{Kontraindikationen}

- Dysplasien des Radiuskopfs oder des Caput radii. Diese werden meist bei über eine längere Zeitspanne (in der Regel > 6-8 Monate) bestehender Luxation beobachtet und müssen auch radiologisch gesucht werden.

\section{Patientenaufklärung}

- Offene Aufklärung der Eltern über mögliche Ursachen der übersehenen Monteggia-Läsion sowie über den prognostisch wichtigen Zeitfaktor.

- Allgemeine Operationsrisiken.

- Notwendigkeit einer Ulnaosteotomie und evtl. einer Eröffnung des Gelenks. gelenks und Unterarms. Das Behandlungsziel hängt wesentlich vom Alter der Verletzung ab [4, 13]. Frische Verletzungen können zu einer Restitutio ad integrum geführt werden [9]. 6-8 Monate alte Verletzungen lassen sich mit Hilfe einer Ulnaosteotomie vollständig zur Ausheilung bringen. Bei > 1 Jahr alten Verletzungen ist nicht mehr mit einer vollständigen Wiederherstellung, sondern nur mit einer Besserung zu rechnen.

- Tragen und Pflege des Fixateur; empfehlenswert ist die Abgabe eines entsprechenden Merkblatts oder einer Broschüre.

- Heilungsdauer der Osteotomie.

- Möglichkeit eines Verfahrenswechsels auf eine Plattenosteosynthese, um die Tragdauer des Fixateurs zu verkürzen.

- Implantatentfernung.

- Notwendigkeit einer engmaschigen Nachbetreuung.

- Physiotherapie.

- Heilungsverzögerung bis hin zur Pseudarthrose.

- Möglichkeit einer erneuten Dislokation.

- Vor allem bei offenem Vorgehen funktionelle Verschlechterung durch Vernarbungen im Gelenk möglich.

- Remodellierungspotenz.

\section{Operationsvorbereitungen}

- Routinelabor.

- Aktuelles präoperatives Röntgenbild.

- Ein CT mit dreidimensionaler Rekonstruktion kann von Vorteil sein, ist jedoch nicht unbedingt notwendig.

- Durchleuchtungsmöglichkeit intraoperativ.

- Intubationsnarkose mit Relaxation erleichtert das Manipulieren der Fragmente nach Osteotomie.

- Genaue Analyse der Verletzung.

- Planung der Lage der Pins und der möglichst proximalen Ulnaosteotomie, am besten mit einer Zeichnung (eine Osteotomie im mittleren Schaftbereich sollte wegen der mangelhaften Korrekturwirkung auf den Radiuskopf vermieden werden).

- Rekonstruktion des Ligamentum anulare notwendig. Eine Rekonstruktion sollte 6-8 Monate nach der verpassten Luxation nicht mehr versucht oder angestrebt werden!

- Bei Anwendung des Ringfixateurs zusätzlich: Montage des Ringsystems, bestehend aus vier Ringen oder 5/8-Ringen, gemeinsam mit Eltern und Kind, um diese damit vertraut zu machen. 


\section{Instrumentarium und Implantate}

- Orthopädisch-traumatologisches Grundinstrumentarium.

- Kleiner AO-Fixateur externe (Fa. Synthes, Abbildung 2a):

- Kombinierbare „click-on clamps“, 4 mm.

- Verbindungsstangen aus Carbon oder Stahl, 4 mm.

- Sel-Drill-Schanz-Schrauben, 2,5/4 mm, 3,0/4 mm und 4,0/4 mm.

- Ringfixateur normal und klein (Fa. Synthes oder Smith \& Nephew, Abbildung 2b):

- Ringe verschiedener Durchmesser.

- Gewindestangen verschiedener Längen.

- Gelenke.

-1,6-mm- bzw. 1,8-mm-Kirschner-Drähte zum Spannen.

- Sel-Drill-Schanz-Schrauben, 2,5/4 mm, 3,0/4 mm und 4,0/4 $\mathrm{mm}$.

- Spanngerät für Kirschner-Drähte.

\section{Anästhesie und Lagerung}

- Intubationsnarkose.

- Rückenlagerung.

- Röntgenstrahlendurchlässiger Armtisch mit freier Durchleuchtungsmöglichkeit (Abbildung 3a).

- Wir bevorzugen wegen des besseren Bilds, des gröBeren Bildausschnitts, der genaueren Durchleuchtungseinstellung und der dadurch kürzeren Durchleuchtungszeit eine Lagerung des Arms direkt auf dem Bildwandler (Abbildung 3b).

- Desinfektion des Arms bis zur Axilla und sterile Abdeckung.

- Bereithaltung einer sterilen Blutsperre, die nur im Fall einer Arthrotomie verwendet wird.

- Immer ohne Blutsperre osteotomieren, um eine Blutung zu erkennen und ein Kompartmentsyndroms zu verhindern.

\section{Abbildungen $2 a$ und $2 b$}

a) Kleiner AO-Fixateur externe, als „,tubeto-tube"-System zu verwenden (Synthes).

b) Kleiner Ringfixateur (Smith \& Nephew).
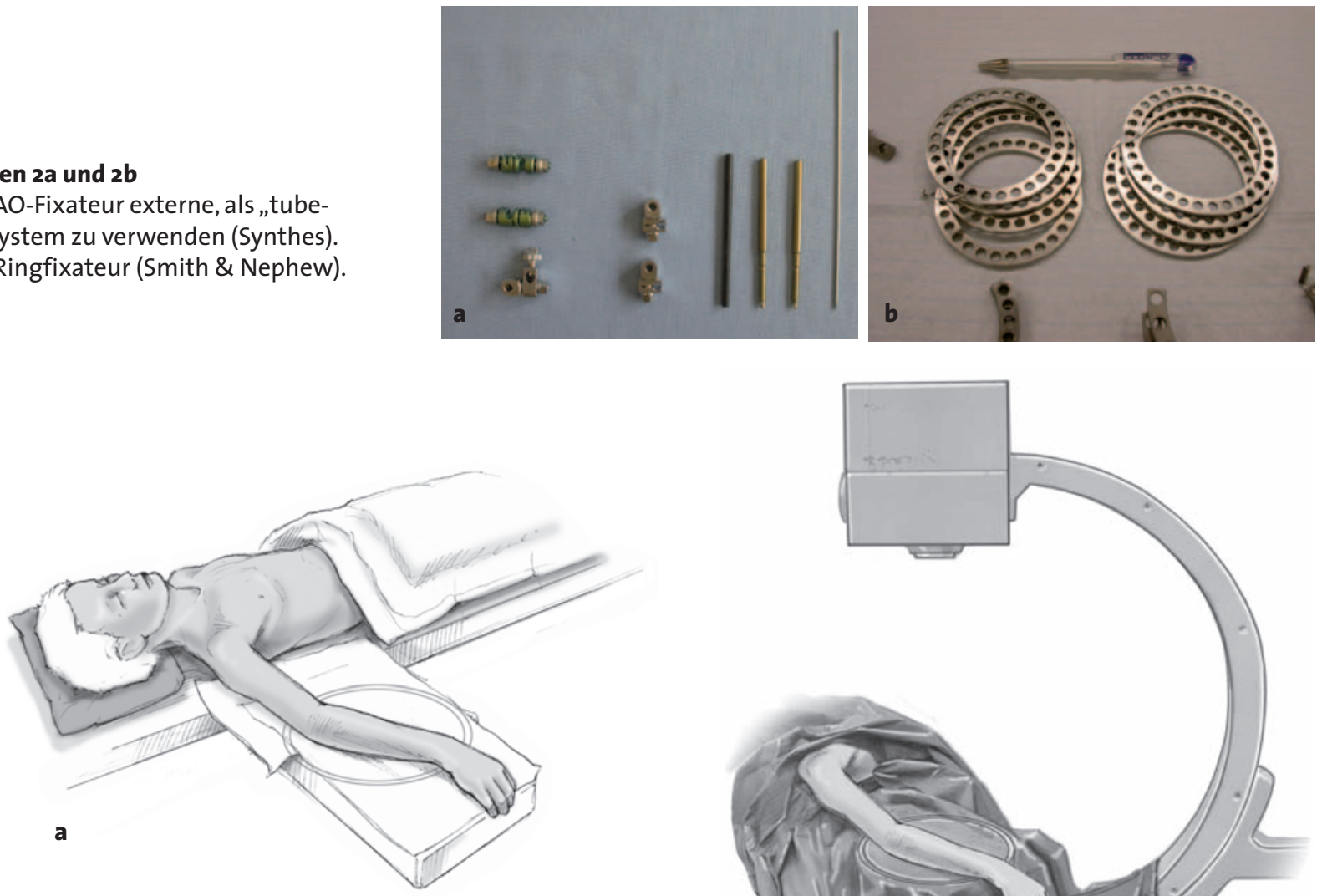

\section{Abbildungen $\mathbf{3 a}$ und $\mathbf{3} \mathbf{b}$}

a) Lagerung des Arms auf einem röntgendurchlässigen Armtisch.

b) Direkte Lagerung des Arms auf dem steril abgedeckten Bildverstärker.

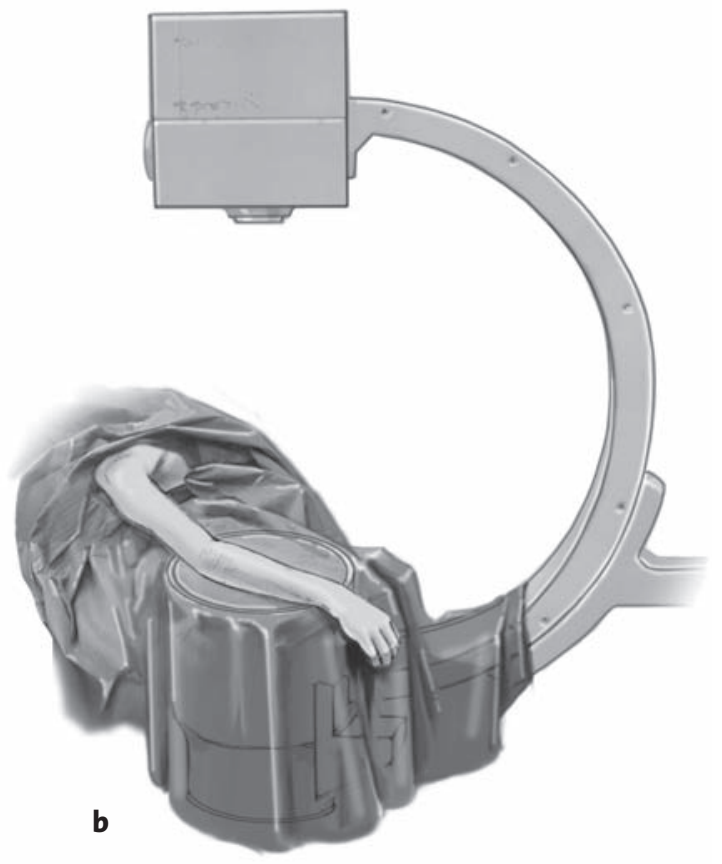




\section{Operationstechnik}

Abbildungen 4 bis 17

\section{Behandlung einer kurzzeitig übersehenen Luxation}

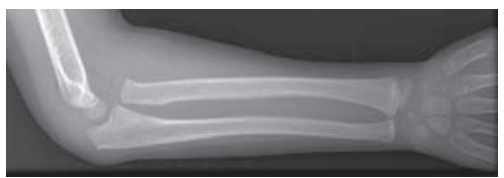

\section{Abbildung 4}

9-jähriges Kind, 6 Wochen nach einer Monteggia-Verletzung mit verheilter Ulnafraktur und luxiertem Radiuskopf nach Sturz von der Schaukel. Angabe von mäßigen Schmerzen bei Flexion und Extension, eingeschränkte Pronation/Supination $\left(70 / 0 / 75^{\circ}\right)$

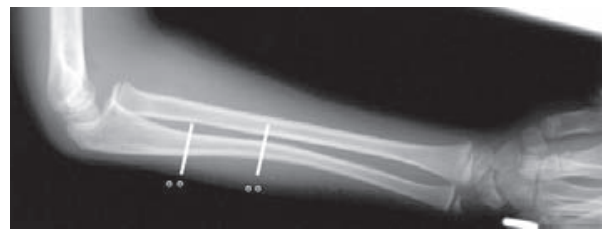

a
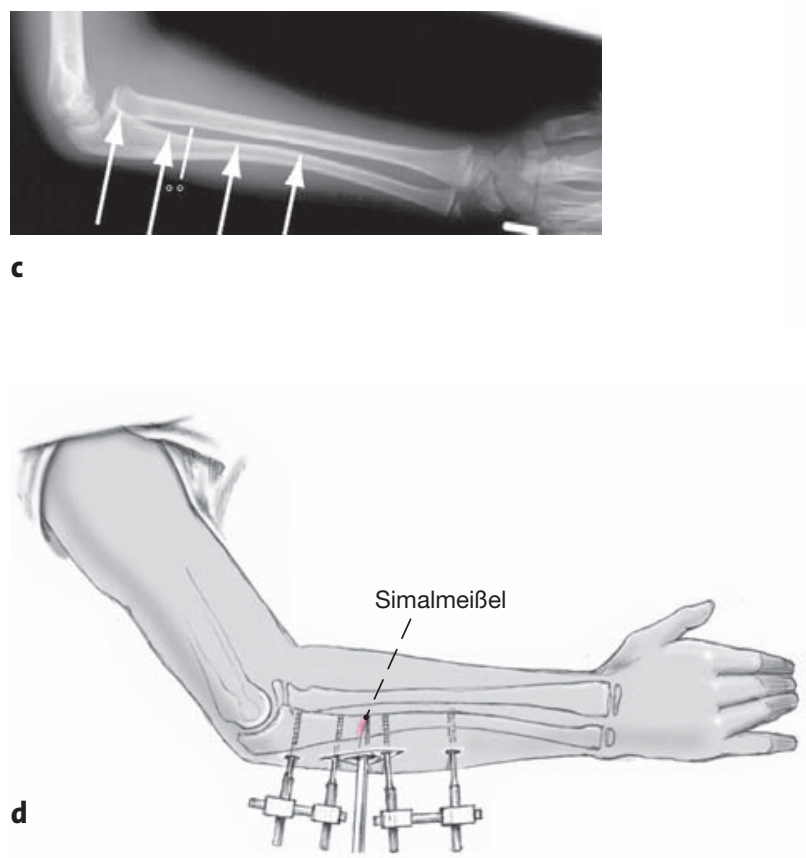

Abbildungen $\mathbf{5 a}$ bis $\mathbf{5 g}$

(Legende s. nächste Seite)

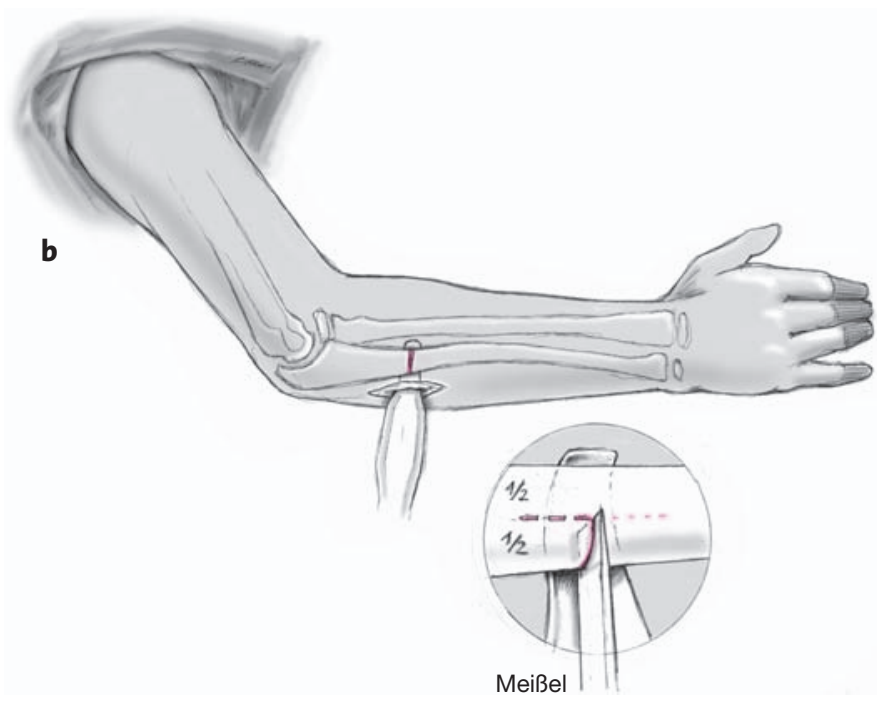

e

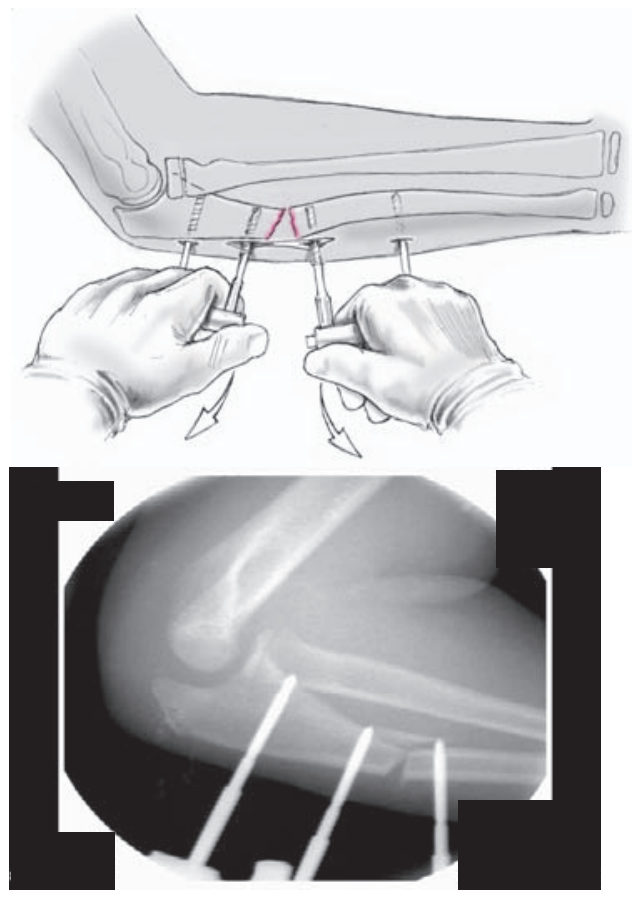



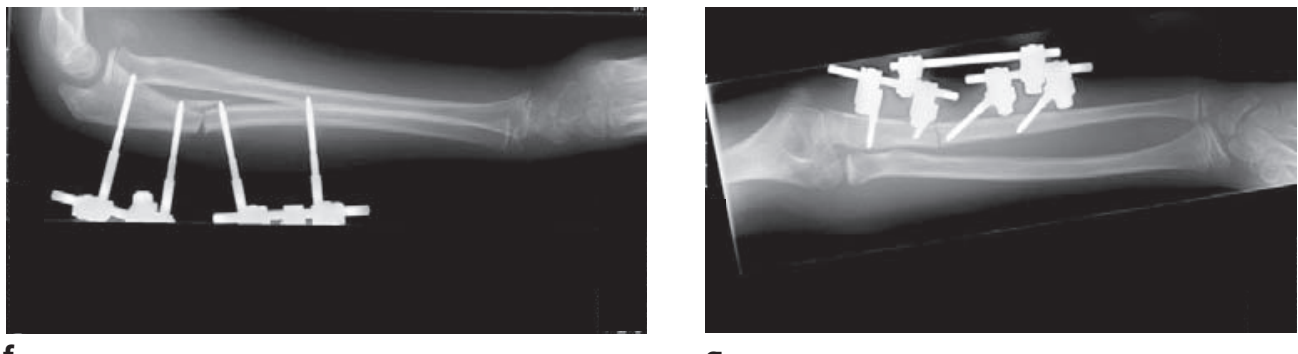

$\mathbf{g}$

\section{Abbildungen 5 a bis $\mathbf{5 g}$}

Unter Durchleuchtung gemäß Planung Festlegung der Osteotomiestelle (a). Etwa $3 \mathrm{~cm}$ lange Hautinzision über der entsprechenden Stelle der Ulna, direktes Eingehen auf das Periost und Inzision desselben, subperiostales Umfahren der Ulna. Die Elle wird nun mit einem scharfen Meißel an der gewünschten Stelle zu ca. 50\% durchtrennt (b).

Platzierung von Schanz-Schrauben je $1 \mathrm{~cm}$ proximal und distal der vorbereiteten Osteotomie (c). Über zwei kleine Inzisionen ca. 3-4 cm proximal und distal der beiden zuvor eingebrachten Schanz-Schrauben Setzen je einer weiteren. Verbinden der beiden proximalen und distalen Schanz-Schrauben mit einer 4-mm-Stange.

Mit einem sog. Simalmeißel (d) vervollständigen wir die Osteotomie. Mit Hilfe der zuvor gesetzten und untereinander verbundenen Schanz-Schrauben Durchbrechen des Knochens von Hand (e).

Durch Fassen der beiden Fixateurteile kann nun die Ulna flektiert und anguliert werden, so dass über Zug an der Membrana interossea der Radius und der Radiuskopf in die korrekte Position einschnappen können. Gleichzeitig wird die Ulna um einige Millimeter verlängert (f, g). Der Radiuskopf muss sich „spontan“ reponieren und in reponierter Stellung bleiben. Verbinden des Fixateurs „tube-to-tube“ mit vorbereiteter Stange.

Unter Durchleuchtung überprüfen wir nun die Reposition in Flexion, Extension, Pronation und Supination. Bei ungenügender Reposition Planung einer kleinen Arthrotomie (s. Abbildungen 6a bis 6c). Anschließend Verschluss des Periosts mit Vicryl $3 / 0$. Subkutannaht, intrakutane Hautnaht mit Vicryl rapid 4/o.

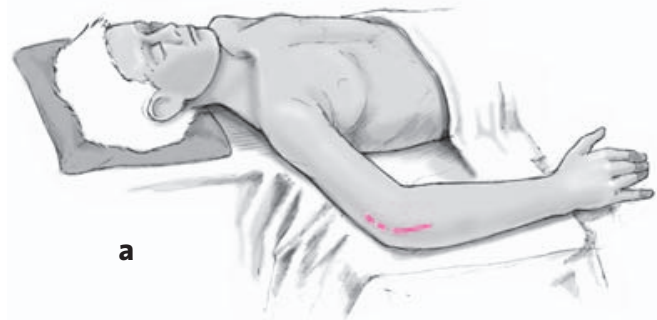

Abbildungen 6a bis $6 \mathrm{c}$

Arthrotomie bei ungenügender Reposition des Radiuskopfs. Radialer, eher dorsal/proximal gelegener Zugang über ca. $4 \mathrm{~cm}$ langen Hautschnitt (a). L-förmiges Ablösen der Extensorenansätze auf einer Länge von ca. $1 \mathrm{~cm}$ und Arthrotomie direkt über dem Caput radii (b). In der Regel sieht man auf das interponierte Ligamentum anulare, das nicht durchtrennt werden darf! Man sucht nun mit einem stumpfen Instrument die Öffnung, durch die der Radiuskopf „durchgeschlüpft“ ist, und weitet diese auf. Durch die Öffnung sieht man auf die Gelenkfläche des Radiuskopfs. Durch „walkende“ Bewegungen und mit Hilfe eines Elevatoriums kann das Ligamentum anulare über den Radiuskopf gestülpt oder der Radiuskopf durch die Öffnung gedrückt werden (c). Der Radiuskopf artikuliert nun wieder mit dem Caput radii, was visuell überprüft wird. Funktionsund Stabilitätsprüfung. Verschluss der Kapsel mit Vicryl 3/o. Readaptation der Muskelansätze mit Vicryl 2/o. Subkutannaht und Hautnaht.
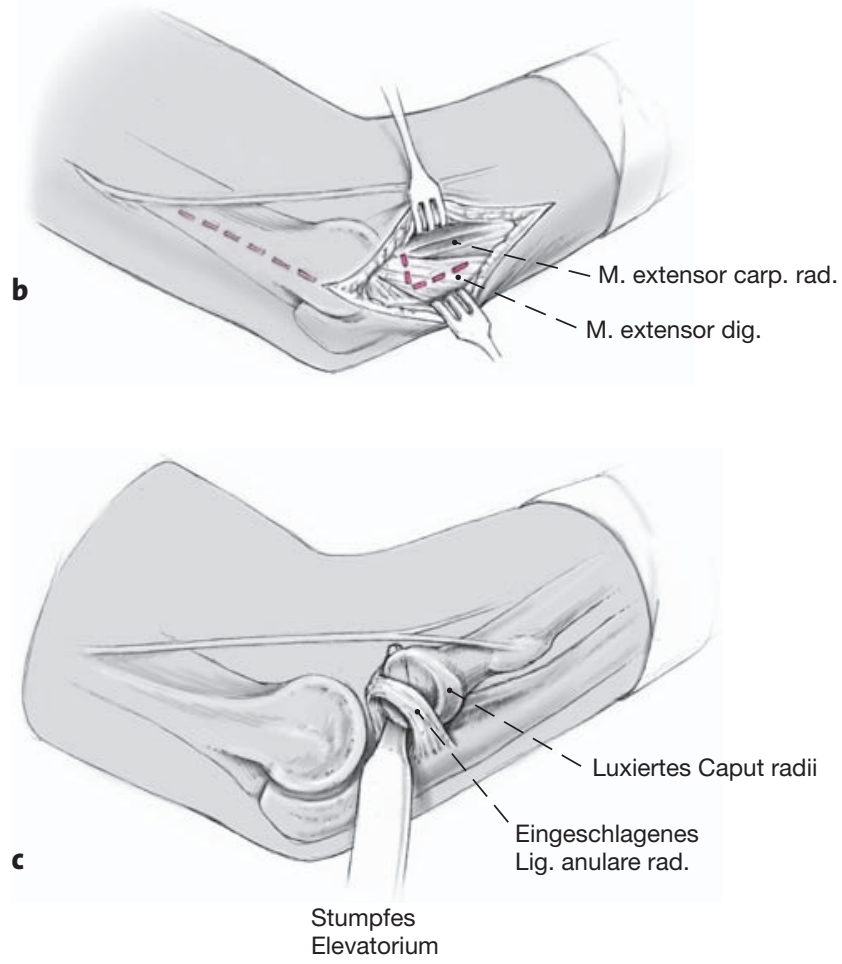

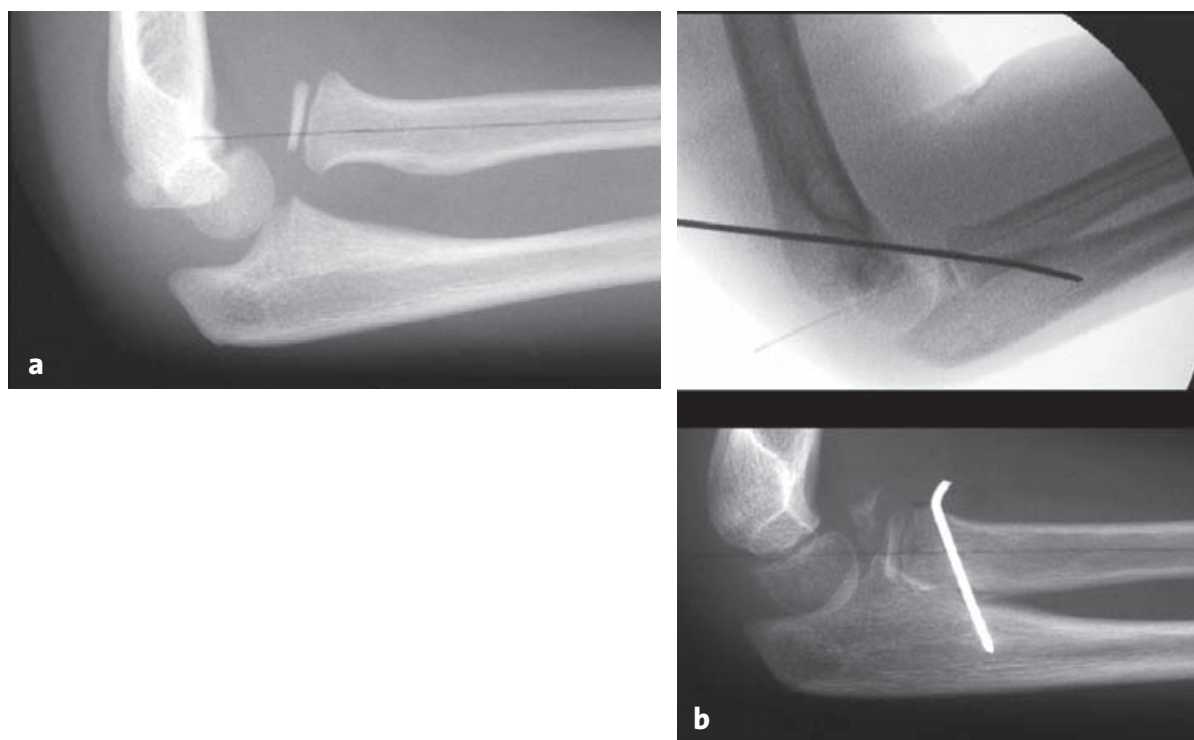

\section{Abbildungen $7 \mathrm{a}$ bis $7 \mathrm{~d}$}

Oft wird die Ulna nur ungenügend verlängert. Dadurch entsteht ein zu hoher Druck auf den Radiuskopf, er reluxiert. Muss der Radiuskopf mit einem Kirschner-Draht gehalten werden,

\section{Behandlung einer über längere Zeit ( $<1$ Jahr ) übersehenen Luxation (Intermediärtyp, s. Abbildung 1a)}

Bei diesem Typ handelt es sich um eine chronische Luxation 4-12 Monate nach dem Unfallereignis. Das proximale Radioulnargelenk hat sich bereits verändert, der Radiuskopf überragt das Capitulum humeri um $>5 \mathrm{~mm}$. Es bestehen eine deutliche Bewegungsbeeinträchtigung und eine verbliebene Fehlstellung der Ulna mit abgeschlossenem Remodeling.

Als erster Schritt müssen, wie oben beschrieben, die Fehlstellung der Ulna beseitigt und eine gegenläufige reponiert bleiben, nach Entfernung eines fixierenden Kirschner-Drahts kommt es in den allermeisten Fällen sofort zur Redislokation $(a-d)$. ist die Reposition ungenügend. Der Radiuskopf muss spontan
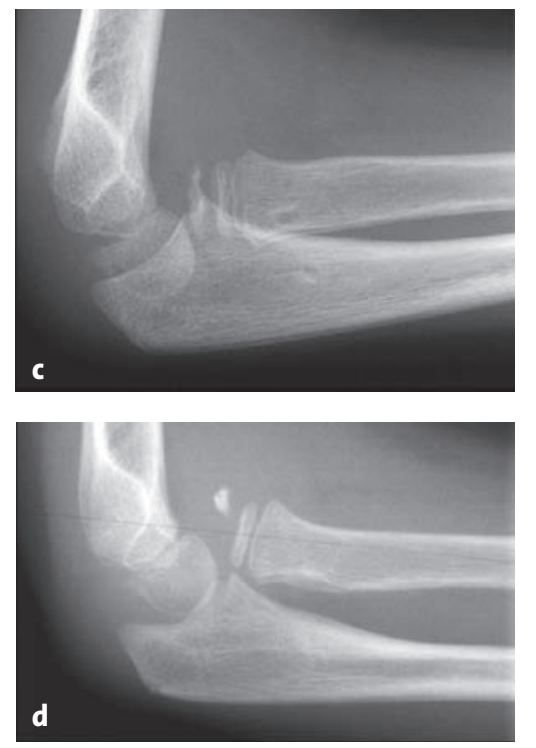


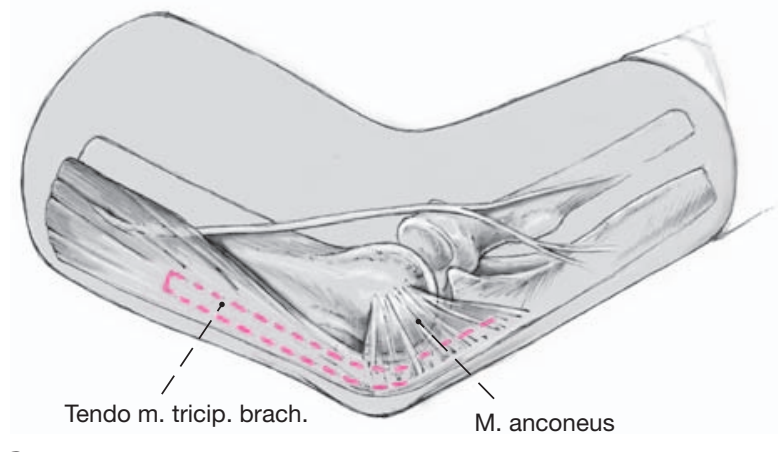

\section{Abbildungen 8a und $8 b$}

Im Folgenden soll zusätzlich nur dieses Vorgehen beschrieben werden, die übrigen Schritte wurden bereits zuvor dargestellt. Erweiterung der in Abbildung 6 a dargestellten Inzision nach dorsal und distal. Der später zu präparierende Faszienstreifen ist auch bereits eingezeichnet (a). Präparation über der Ulnakante unter Belassung des Periosts und einiger Muskelfasern des Musculus anconeus [8] (wie in Abbildung 6b). Anheben des

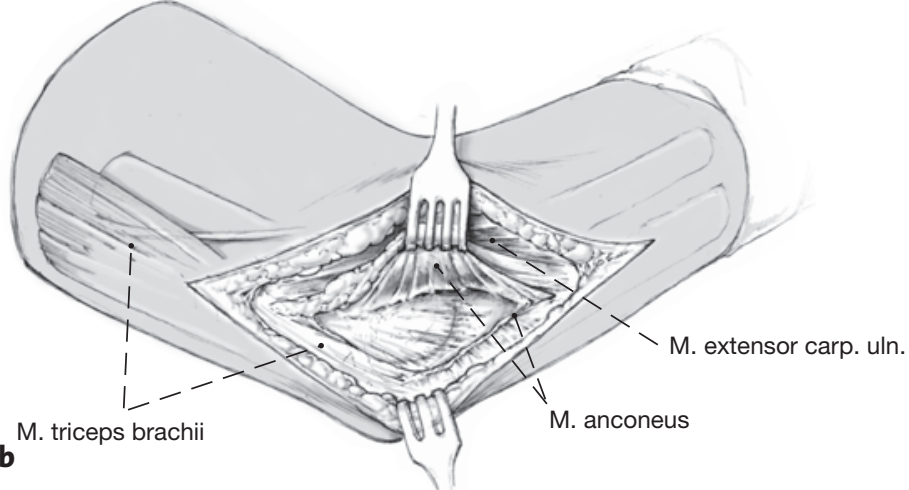

ganzen Muskels, um von dorsal an das Radioulnargelenk zu kommen (b). Es darf nicht zu distal präpariert werden, da sonst die Gefahr einer Verletzung des tiefen Asts des Nervus radialis besteht, welcher dort durch den Supinatorkanal verläuft. Vom lateralen Aspekt des Capitulum humeri nach distal Eröffnung des Gelenks. Ausräumung des narbig veränderten Rests des Ligamentum anulare.

\section{Abbildung 9}

Darstellung der Trizepssehne und Ausschneiden eines distal am Periost gestielten, $1 \mathrm{~cm}$ breiten und mindestens $5 \mathrm{~cm}$ langen Faszienstreifens. Abpräparieren des Streifens von der Muskulatur; es muss darauf geachtet werden, dass eine feste Verbindung distal zum Periost bestehen bleibt.

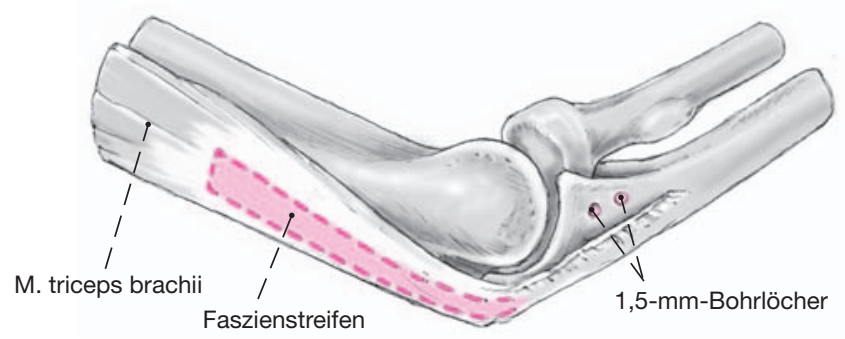

\section{Abbildung 10}

An der freien Ulnakante werden zwei 1,5-mm-Bohrlöcher gesetzt.

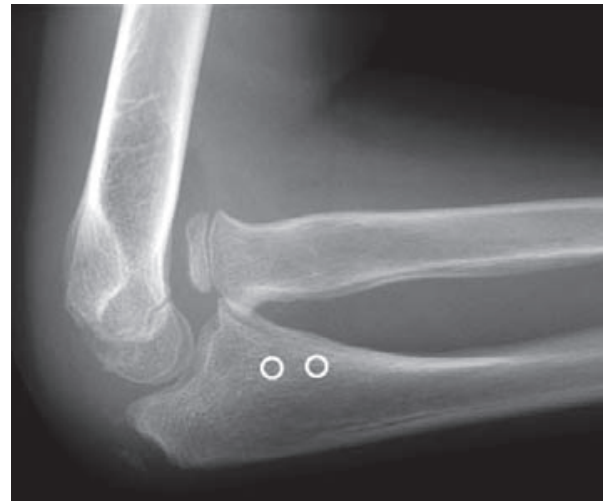




\section{Abbildung 11}

Nach definitiver Reposition des Radiuskopfs durch Verlängerung und Angulation der Ulna führen wir den Faszienstreifen um den Radiushals herum. Sein Ende wird mit PDS-Nähten der Stärke 1/o transossär durch die vorbereiteten Bohrlöcher fixiert. Stabilitäts- und Funktionsprüfung. Wundverschluss.

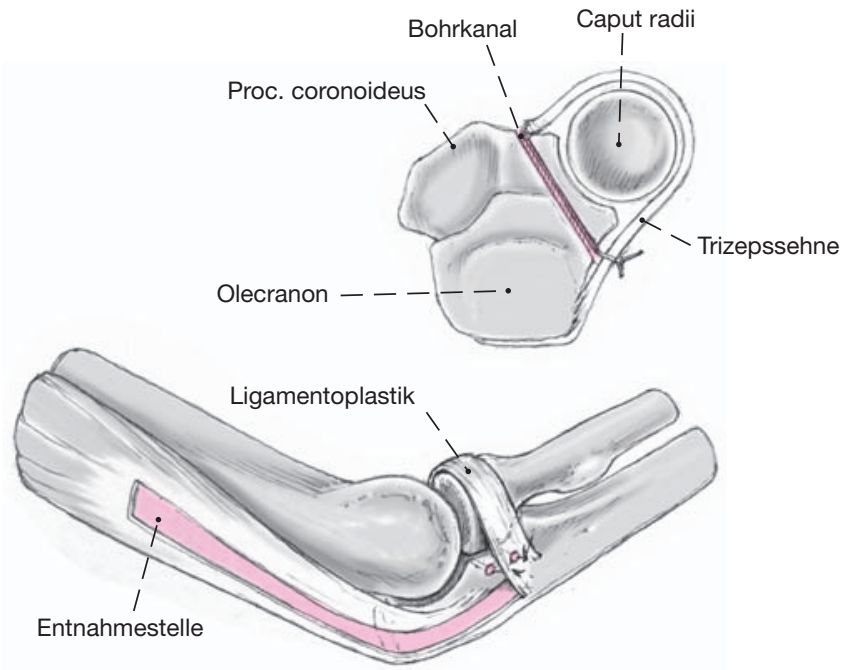

der Behandlung dieser Verletzung hat, ist es wichtig, nur die Dislokation des Radius zu beseitigen und den Radiuskopf genügend nach distal zu bringen. Eine sekundäre, offene Einstellung wird nicht empfohlen und sollte auch nicht versucht werden. Dies entspricht auch unseren eigenen Erfahrungen [5, 12].

\section{Abbildung 12}

Bei diesem Typ handelt es sich um eine Luxation mit einer Dauer über Jahre und einem komplett veränderten proximalen Radioulnargelenk. Der Radiuskopf überragt das Capitulum humeri um 2-3 $\mathrm{cm}$. Es bestehen eine massive Bewegungsbeeinträchtigung sowie zunehmende Beschwerden und Schmerzen. Das Remodeling der verbliebenen Fehlstellung der Ulna ist abgeschlossen.

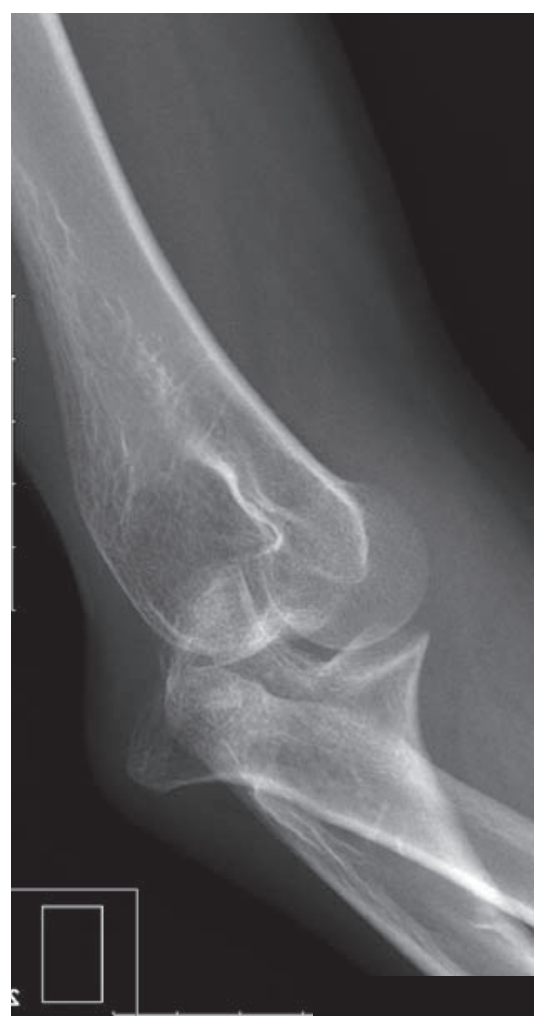




\section{Abbildung 13}

Ziel der Behandlung ist es, eine Situation zu schaffen, die einer Resektion des Radiuskopfs gleichkommt, ohne diesen jedoch zu resezieren und die entsprechenden Nachteile in Kauf nehmen zu müssen. Häufig kommt es zu einer spontanen teilweisen bis vollständigen Reposition des Kopfs.
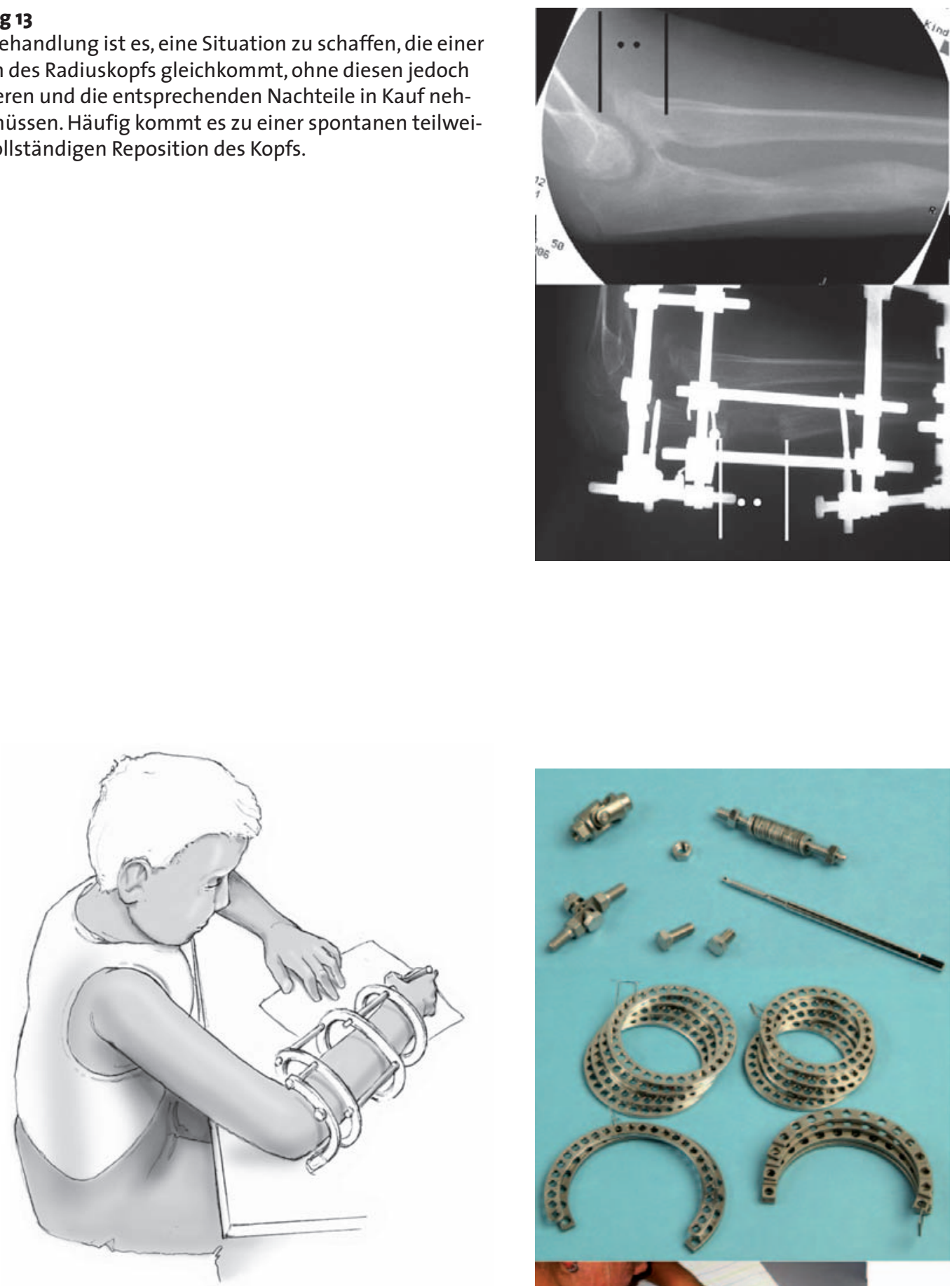

\section{Abbildung 14}

Unsere Erfahrung hat gezeigt, dass monolaterale Systeme dieses Problem nur ungenügend oder gar nicht lösen können. Distal müssen Radius und Ulna gefasst werden, um eine simultane Distalisation des Radius mit der unter Verlängerung stehenden Ulna zu erreichen. Die freie Platzierung von Drähten beim Ringsystem erleichtert dies. Kenntnisse in der Technik der Knochenverlängerung sollten vorhanden sein.

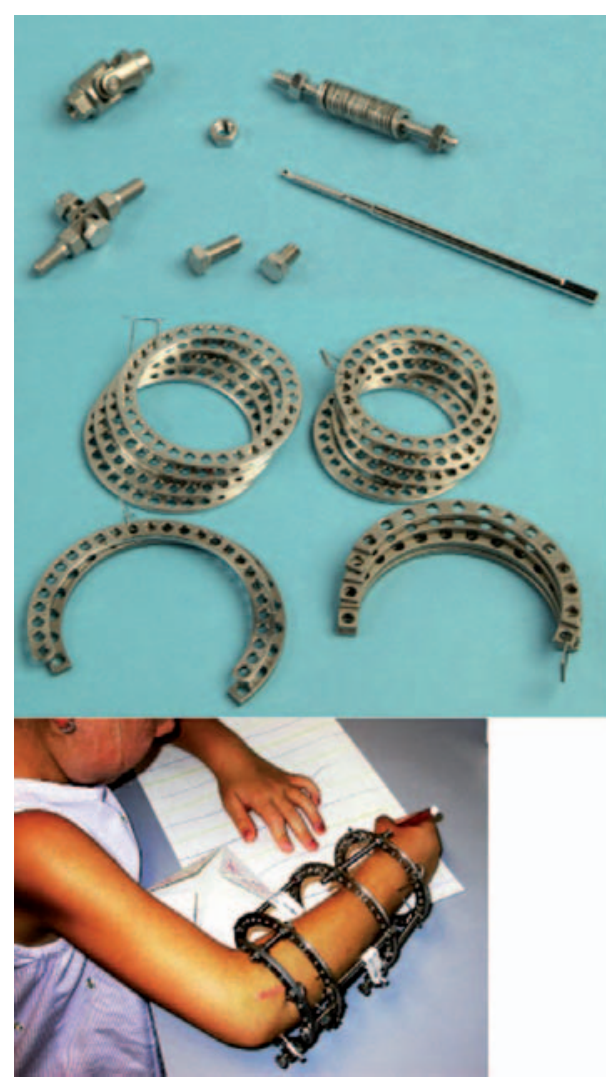




\section{Abbildung 15}

$3 \mathrm{~cm}$ lange, längs verlaufende Inzision über der Ulnakante und subperiostales Umfahren der Ulna an der zuvor mit dem Bildwandler festgelegten Osteotomiestelle. Mit dem 8-mm-Simalmeißel wird die Ulna zu zwei Dritteln osteotomiert, ohne sie zu brechen. Das vormontierte Ringsystem wird über den Arm gestülpt. Genaue Platzierung des distalsten Rings oder 5/8-Rings auf Höhe des Processus coronoideus und Fixierung mit einem Spanndraht. Genaues Ausrichten des Rings und des Arms innerhalb der Ringe, so dass Arm und Handgelenk genau im Zentrum liegen. Am distalsten Ring ca. $2 \mathrm{~cm}$ proximal der distalen Ulnafuge Anbringen und Spannen des zweiten Drahts. Damit sind der Arm fixiert und der Apparat ausgerichtet.

\section{Abbildung $\mathbf{1 6}$}

Proximaler Ring 1: Zusätzlichen „half-pin“

(= Schanz-Schraube) $3 \mathrm{~mm}$ auf Ulnakante eindrehen. Proximaler Ring 2 (proximal der Osteotomie): Ebenfalls mit Draht und Schanz-Schraube fixieren.

Distaler Ring 3 (distal der Osteotomie): Fixierung wie Ring 2.

Distalster Ring 4: Zusätzlich Radius mit Spanndraht fassen, so dass keine neurovaskulären Strukturen verletzt werden (dieser Draht zieht den Radius nach vorn). Vervollständigung der Osteotomie mit dem Meißel und radiologische Kontrolle unter dem Bildwandler. Verschluss der kleinen Wunde mit Vicryl rapid 4/o. Anlegen eines lockeren Verbands.
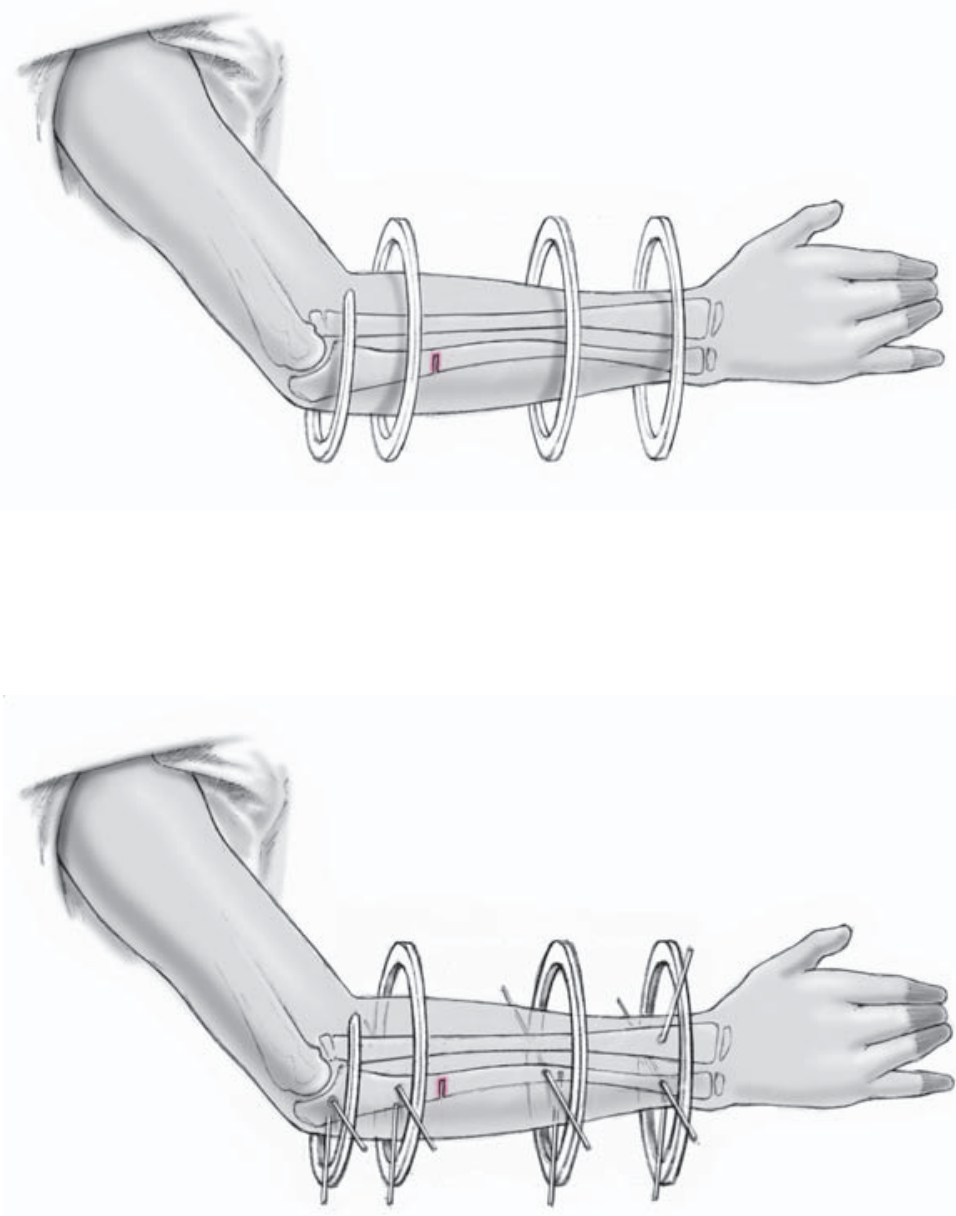


\begin{abstract}
Abbildungen 17a bis $17 \mathrm{c}$
Anpassungen.

Der Radiuskopf muss mindestens $1 \mathrm{~cm}$ distal des

Capitulum humeri zu liegen kommen, um eine freie und schmerzlose Flexion/Extension, bestenfalls auch Umwendbewegung erreichen zu können.

Eine gute knöcherne Konsolidierung muss vor Abnahme des Fixateurs unbedingt abgewartet werden; d.h., eine sog. Kortikalisation muss sichtbar sein.

Ambulante Fixateurentfernung in sog. Kalinox (= Lachgasnarkose) oder konventioneller Kurznarkose. Danach empfiehlt es sich, für einige Tage bis 2 Wochen eine Schutzschiene zu tragen.

a) Darstellung des Ringsystems vor Beginn der Distraktion, distal ist der Radius mitgefasst. Man beachte noch die hoch kubitale Lage des Radiuskopfes (oberes Bild). Nach Distraktion von mehr als $2 \mathrm{~cm}$ liegt nun der Radiuskopf deutlich distal des Capitulum humeri (unteres Bild). b) Schon fortgeschrittener Durchbau der Osteotomie nach 4 Wochen. Auf Grund der Überdistraktion kann der Radiuskopf praktisch spontan in die korrekte Lage, zentriert auf das Capitulum humeri rutschen.

c) Nach völliger Konsolidation und Abnahme des Fixateurs perfekte, spontane Reposition und Zentrierung des Radiuskopfes sowie weitgehend normale Funktion für Beugung und Streckung; die Umwendbewegung ist in beiden Richtungen 30\% eingeschränkt.
\end{abstract}

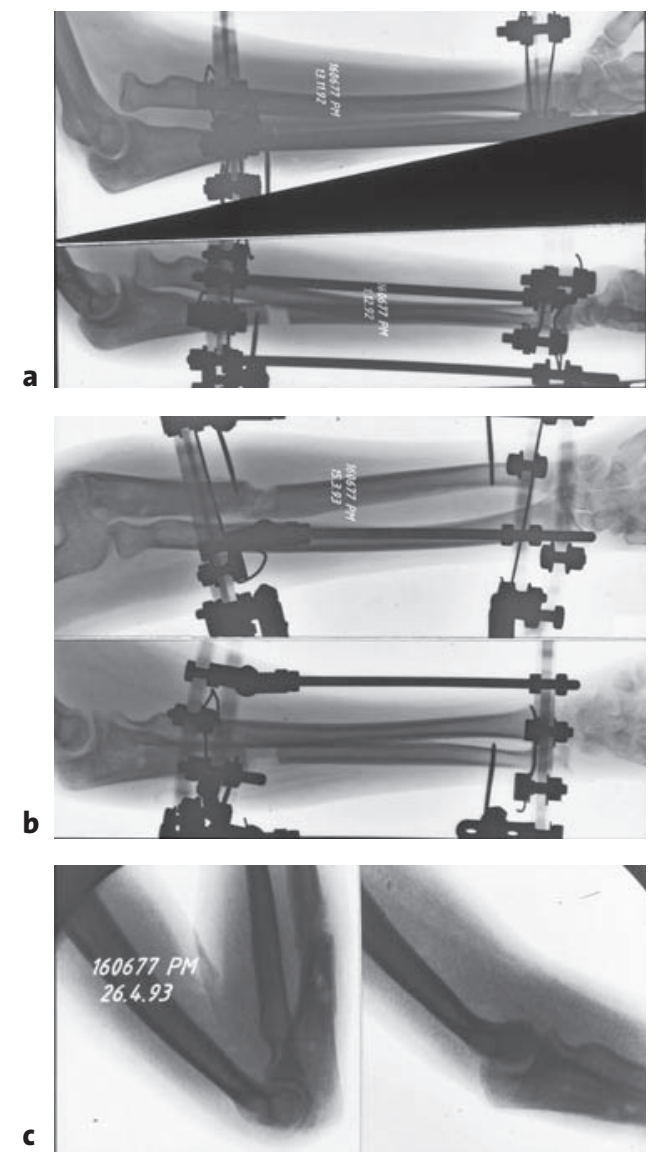

Nach Rekonstruktion des Ligamentum anulare

- Je nach Alter und Kooperation des Kinds kann es notwendig sein, für 3-4 Wochen einen funktionellen, d.h. abnehmbaren Gipsverband anzulegen, um eine ungestörte Einheilung des Ligaments zu erreichen.

- Sport nach 2-3 Monaten möglich.

\section{Bei Anlage eines Ringfixateurs}

- Neurovaskuläre Überwachung für 24 h.

- Distraktion ab dem 5. postoperativen Tag $1 \mathrm{~mm}$ pro Tag $(4 \times 1 / 4 \mathrm{~mm})$.

- Entlassung in der Regel am 6. Tag.

- Keine prophylaktische Antibiotikagabe.

- Beginn einer Physiotherapie, um Beugekontrakturen im Ellbogengelenk vorzubeugen.

- Klinische Kontrollen alle 2-3 Wochen.

- Radiologische Kontrolle bei Erreichen der geplanten Verlängerung. 


\section{Abbildungen 18a bis 18d}

a) Situation bei veralteter Radiuskopfluxation ( $>1$ Jahr).

b) Versuch der Ulnaverlängerung ohne gleichzeitige Fixierung des Radius distal mit monolateralem Fixateur externe; man beachte, dass der Radiuskopf nicht distalisiert wird.

c) Nach Abnahme des Fixateur externe verbleibende Radiuskopfluxation.

d) Nochmalige Verlängerung mit Ringfixateur; man beachte die suffiziente Wanderung des Radius und die schöne spontane Einstellung des Kopfs.
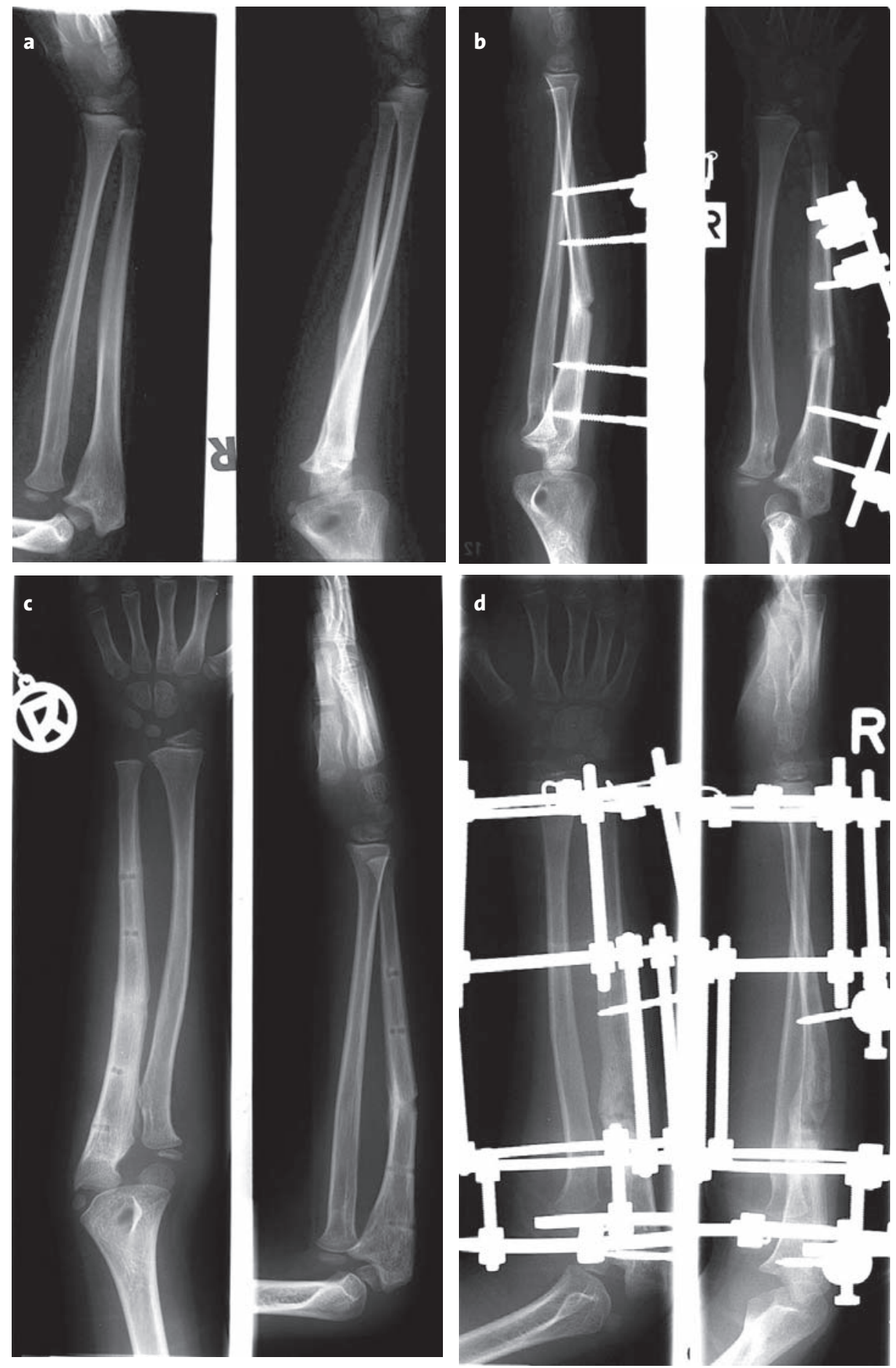

- Anpassung der Distraktion je nach Stand des Radiuskopfs.

- Überwachung der Physiotherapie.

Fehler, Gefahren, Komplikationen

- Ungenügende Verlängerung der Ulna: Muss diese $>5 \mathrm{~mm}$ verlängert werden, fixieren wir Radius und Ulna distal temporär mit einem Kirschner-Draht, um den Verlängerungszug von der Ulna auch auf den Ra- 
dius zu übertragen. Anderenfalls wird der Verlängerungszug durch die Membrana interossea aufgehoben!

- Infektion der Pineintrittsstellen: Bessere Reinigung, orale Antibiotika (Cefuroxim, Clindamycin, Amoxicillin + Clavulansäure).

- Persistenz oder Zunahme der Entzündung über 48 h: Hospitalisation und intravenöse Antibiotikagabe.

\section{Bei Verwendung des Ringfixateurs}

- Radiuskopf steht trotz korrekter Länge noch zu hoch: Einbau eines Gelenks zur Angulation, evtl. kombinierte Angulation und weitere Distraktion.

- Adaptation der Ringe bei schlechtem Alignment ohne Narkose möglich.

- Konsolidierungsstörung: 1.zyklische Kompression/Distraktion, 2. Magnetfeldtherapie, 3. Injektion von Knochenmark, 4. minimalinvasive Plattenosteosynthese.

- Reluxationen sind oft kaum mehr zu behandeln. Sie erfordern aufwendige Rekonstruktionen, oft auch ein offenes Vorgehen. Die Funktion wird dadurch meist schlechter; dies ist nicht durch Zahlen belegt, geht aber aus der eigenen Erfahrung hervor.

\section{Ergebnisse}

\section{Nach kurzzeitig übersehener Luxation}

Bei korrekter Indikationsstellung und Planung sowie oben beschriebener Behandlung sind die Ergebnisse durchaus gut. Prinzipiell gibt es bei einer spontanen oder spontan gehaltenen Einstellung keinen Grund für eine Redislokation. Die Angaben in der Literatur sind widersprüchlich, dies jedoch meist wegen insuffizienter Behandlungen, Fehlens von geeignetem Equipment und falscher Indikation $[3,6,9,11]$.

\section{Nach über eine längere Zeit ( $<1$ Jahr ) übersehener Luxation}

Das Ergebnis bei diesem Typ wird weitgehend durch die sekundäre Deformation des Radiuskopfs wie auch des proximalen Radioulnargelenks bestimmt. Letztere wird anfangs oft übersehen oder unterschätzt. Bei korrekter Indikationsstellung und Planung sollten die Ergebnisse jedoch durchweg gut sein. Dennoch darf es nicht überraschen, wenn während einer recht langen Zeit die Funktion bezüglich Flexion wie Extension, aber auch Pronation und Supination eingeschränkt bleibt. Redislokationen sind beschrieben und entsprechen auch den eigenen Erfahrungen [11,13].

\section{Nach chronischer, über Jahre bestehender Luxation} Das Ergebnis bei diesem Typ wird weitgehend durch die vorbestehenden Probleme sowie den verwendeten
Fixateur (monolateraler Fixateur vs. Ringfixateur, Abbildungen 18a bis 18d) beeinflusst: Häufig findet man vorbestehende Bewegungseinschränkungen, Muskelverkürzungen sowie Schmerzen und Neuropathien.

Eine ungenügende Verlängerung löst das Problem oft nicht, ein zusätzlicher offener Repositionsversuch führt nicht selten zu einer Verschlechterung der Ausgangslage. Es soll nochmals darauf hingewiesen werden, dass die Indikation für eine solche Behandlung gut überlegt werden muss und zurückhaltend gestellt werden sollte. Zudem muss vor zu hohen Erwartungen gewarnt werden $[5,10,12]$.

\section{Literatur}

1. Bado JL. The Monteggia lesion. Springfield:Thomas, 1962.

2. David-West KS, Wilson NI, Sherlock DA, et al. Missed Monteggia injuries. Injury 2005;36:1206-9.

3. Degreef I, De Smet L. Missed radial head dislocations in children associated with ulnar deformation: treatment by open reduction and ulnar osteotomy. J Orthop Trauma 2004;18:375-8.

4. Fowles JV, Sliman N, Kassab MT. The Monteggia lesion in children. Fracture of the ulna and dislocation of the radial head. J Bone Joint Surg Am 1983;65:1276-82.

5. Gicquel P, De Billy B, Karger C, et al. [Treatment of neglected Monteggia's fracture by ulnar lengthening using the Ilizarov technique.] Rev Chir Orthop Reparatrice Appar Mot 2000;86:844-7.

6. Hasler CC, Laer L von, Hell AK. Open reduction, ulnar osteotomy and external fixation for chronic anterior dislocation of the head of the radius. J Bone Joint Surg Br 2005;87:88-94.

7. Hefti F, et al. Kinderorthopädie in der Praxis. Berlin-Heidelberg-New York: Springer, 1998.

8. Morrissy RT, Weinstein SL. Atlas of pediatric orthopedic surgery. Philadelphia:Williams \& Wilkins, 2001.

9. Oner FC, Diepstraten AF. Treatment of chronic post-traumatic dislocation of the radial head in children. J Bone Joint Surg Br 1993;75:577-81.

10. Rodgers WB, Waters PM, Hall JE. Chronic Monteggia lesions in children. Complications and results of reconstruction. J Bone Joint Surg Am 1996;78:1322-9.

11. Seel MJ, Peterson HA. Management of chronic posttraumatic radial head dislocation in children. J Pediatr Orthop 1999;19:306-12.

12. Villa A, Paley $D$, Catagni MA, et al. Lengthening of the forearm by the Ilizarov technique. Clin Orthop 1990;250:125-37.

13. Wang MN, Chang WN. Chronic posttraumatic anterior dislocation of the radial head in children: thirteen cases treated by open reduction, ulnar osteotomy, and annular ligament reconstruction through a Boyd incision. J Orthop Trauma 2006;20:1-5.

\section{Korrespondenzanschrift}

Dr. Theddy Slongo

Leitender Arzt für Kindertraumatologie und Kinderorthopädie Abteilung für Pädiatrische Chirurgie

Universitätskinderklinik

Inselspital

3010 Bern

Schweiz

Telefon (+41/31) 632-9293, Fax -9292

E-Mail: theddy.slongo@insel.ch 